\title{
Efeito da complexidade da tarefa, idade e género na assimetria motora funcional de crianças destrímanas e sinistrómanas
}

\author{
Task complexity, age and gender effect on functional motor asymmetry \\ of right- and left-handed children
}

\author{
P.C. Rodrigues, S.C.M. Carneiro, I. Cabral, M.O. Vasconcelos, J.M. Barreiros
}

\begin{abstract}
RESUMO
A assimetria manual foi analisada na realização de tarefas de antecipação-coincidência de complexidade distinta em 59 crianças destrímanas e 56 sinistrómanas de ambos os sexos, divididas em dois grupos de idade (7-8 anos e 9-10 anos). Os resultados deste estudo revelaram (i) um aumento da assimetria manual com a complexidade da tarefa em ambos os grupos de PM, apesar de nos sinistrómanos a diferença entre mãos não ter atingido significado estatístico; (ii) uma assimetria manual em todos os erros de medida, evidente apenas nos sinistrómanos; (iii) um desempenho mais proficiente da tarefa simples comparativamente à tarefa complexa em ambos os grupos de PM; (iv) um efeito maturacional mais pronunciado na execução da tarefa complexa; (v) maior precisão e menor variabilidade do grupo 9-10 anos comparativamente ao grupo 7-8 anos, em ambos os grupos de PM; (vi) um desempenho mais proficiente do sexo masculino em relação ao sexo feminino em ambos os grupos de PM. Em conjunto, os resultados sugerem que existe uma forte associação entre as características da tarefa e a preferência motora.

Palavras-chave: preferência manual, assimetria motora funcional, complexidade da tarefa, idade, género
\end{abstract}

ABSTRACT

Manual asymmetry was assessed in different complexity coincidence-anticipation tasks in 59 right- and 56 left-handed children of both genders divided into two age groups (7-8 years and 9-10 years). Results revealed that (i) manual asymmetry increased with task complexity in both handedness groups without reaching statistical significance for the left-handed group; (ii) for the left-handed group, manual asymmetry was evident in all measured errors; (iii) better performance was presented when executing simpler tasks for both handedness groups; (iv) maturational effect was more pronounced in the complex task; (v) in both handedness groups, the 9-10-year-old group was more accurate and less variable when compared to the 7-8 year-old group; (vi) interaction between age and task complexity revealed that males outperformed females in both handedness. These results favor the perspective in which handedness is seen as a dynamic process, where motor preference interacts with task complexity.

Keywords: manual preference, functional motor asymmetry, task complexity, age, gender

Submetido: 25.07.2010 | Aceite: 10.12.2010

Paula Cristina Rodrigues, Sara Cristiana Moreira Carneiro, Isabel Cabral e Maria Olga Vasconcelos. Faculdade de Desporto, Universidade do Porto, Portugal.

João Manuel Barreiros. Faculdade de Motricidade Humana, Universidade Técnica de Lisboa, Portugal. Endereço para correspondência: Paula Cristina Rodrigues, Rua da Fonte Branca, nº124, 4415-470 Grijó, V. N.

Gaia, Portugal.

E-mail: packn2@gmail.com 
A assimetria manual, favorecendo a mão preferida (MP) comparativamente à mão não preferida (MNP), é evidente em muitas tarefas do dia-a-dia bem como em tarefas laboratoriais como, por exemplo, toques repetidos com os dedos (Fearing, Browning, Corey, \& Foundas, 2001; Nalcaci, Kalaycioglu, Cicek, \& Genc, 2001; Teixeira \& Paroli, 2000), deslocamento de pregos (Dellatolas et al., 2003; Steenhuis, 1999), pontilhação (Borod, Caron, \& Koff, 1984; Steenhuis, 1999), força de preensão manual (Provins \& Magliaro, 1989; Steenhuis, 1999), antecipação-coincidência (Cockerill, Van-Zyl, \& Nevill, 1988; Coker, 2004; Rodrigues, Vasconcelos, Barreiros, \& Barbosa, 2009; Rodrigues, Vasconcelos, Barreiros, Barbosa, \& Trifílio, 2009), entre outras. Alguns autores atribuíram esta superioridade a assimetrias funcionais hemisféricas associadas aos processos correntes de controlo motor (Haaland \& Harrington, 1996) ou a assimetrias cerebrais estruturais de áreas motoras envolvidas na programação do movimento (Amunts, Jancke, Mohlberg, Steinmetz, \& Zilles, 2000; Triggs, Calvanio, \& Levine, 1997; Volkmann, Schnitzler, Witte, \& Freund, 1998). Para além disso, existe bastante evidência na literatura de que a prática diferenciada das mãos tem implicação específica na dimensão motora de habilidades manuais (Pascual-Leone \& Torres, 1993; Provins, 1997). Parece, desta forma, que as relações entre estruturas neurobiológicas e preferência manual são influenciadas e influenciam, numa dinâmica de reciprocidade, as tarefas e a experiência.

Um dos factores que parece afectar a maior ou menor assimetria funcional é a complexidade da tarefa, tendo os resultados de alguns estudos demonstrado que quanto mais elevada a complexidade da tarefa maior o grau de assimetria. Neste sentido, será possível em tarefas percebidas como mais simples encontrar respostas menos diferenciadas entre o lado esquerdo e o lado direito (Borod et al., 1984; Bryden \& Roy, 1999; Bryden, Roy, Rohr, \& Egilo, 2007; Flowers, 1975; Miller, 1982; Provins \& Magliaro, 1989). No entanto, esta correspondência hipotética entre complexidade e preferência não tem sido encontrada em muitos outros estudos, o que sugere que a natureza perceptivo-motora das tarefas, ou o grau de complexidade sob investigação, podem assumir um papel central nesta questão (Bryden, 2002; Lage et al., 2008; Shen \& Franz, 2005; Teixeira, Gasparetto, \& Sugie, 1999).

Tem sido descrita uma assimetria manual mais acentuada em destrímanos do que em sinistrómanos em várias tarefas, que avaliam o tempo de reacção unimanual (Olex-Zarychta \& Raczek, 2008) e bimanual (Shen \& Franz, 2005), deslocamento de pinos (Bryden, Roy, \& Spence, 2007; Gurd, Schulz, Cherkas, \& Ebers, 2006; Herve et al., 2009), deslocamento de pinos computorizados (Dellatolas et al., 2003; Elalmis \& Tan, 2008) e toques repetidos (Herve, Mazoyer, Crivello, Perchey, \& TzourioMazoyer, 2005; Kumar \& Mandal, 2004; Nalcaci et al., 2001). Em tarefas de antecipação-coincidência (AC) efectuadas com adultos, não foram encontrados efeitos da complexidade da tarefa (Rodrigues, Vasconcelos, Barreiros, \& Barbosa, 2009; Rodrigues, Vasconcelos, Barreiros, Barbosa, \& Trifílio, 2009).

Uma vez que, por volta dos sete anos de idade, a consistência da PM, tanto em destrímanos, como em sinistrómanos, está já determinada (Bryden \& Mayer, 2008; McManus et al., 1988), bem como a capacidade de AC adquirida (Bard, Fleury, \& Gagnon, 1990), pretendeu-se investigar em crianças, e através desta capacidade, o efeito da complexidade da tarefa na assimetria manual. Para além disso, como o desempenho em tarefas de AC revela ser afectado pela variável sexo (Les, Katene, \& Fleming, 2002; Millslagle, 2004; Williams \& Jasiewicz, 2001) esta será igualmente considerada na análise.

\section{Amostra}

\section{MÉTODO}

Participaram no estudo 115 crianças de ambos os sexos (67 do sexo masculino e 48 do sexo feminino), divididos em dois grupos de 
idade (7-8 anos e 9-10 anos). As crianças frequentavam escolas públicas da região do norte de Portugal. Numa fase inicial foram seleccionados os alunos sinistrómanos, tendo como critério a mão preferida para escrever. De seguida, foram escolhidos aleatoriamente alunos destrímanos com características semelhantes aos sinistrómanos, nomeadamente no que respeita ao sexo e à idade. Numa fase posterior, os sujeitos foram novamente avaliados relativamente à sua preferência manual, desta vez de uma forma mais pormenorizada através da aplicação do Dutch Handedness Questionnaire de Van Strien (1992). Este questionário consiste em 15 itens relativos a actividades simples, unimanuais, da vida diária. Para a execução de cada actividade, os sujeitos são solicitados a responder se utilizam a mão direita, a mão esquerda ou se não têm preferência pela utilização de qualquer delas. Cada item é codificado entre 0 e 2, recebendo a mão esquerda o valor de 0 , a direita, o valor de 2 , e qualquer delas, o valor de 1 . Assim, o valor total reporta-se à soma de todos os itens e designa-se por coeficiente de lateralidade, o qual se situa entre 0 e 30 . Os sujeitos foram classificados como sinistrómanos apresentando um coeficiente de lateralidade inferior a 15 ( 24 do sexo feminino e 32 do sexo masculino) e considerados destrímanos com um valor superior a 15 (24 do sexo feminino e 35 do sexo masculino). A média do coeficiente de lateralidade foi de 27.8 \pm 2.8 para os destrímanos (sexo masculino: $27.7 \pm 3.1$, sexo feminino: $28.0 \pm 2.2$ ) e de 4.9 \pm 3.8 para os sinistrómanos (sexo masculino: $4.4 \pm 3.4$, sexo feminino: $5.6 \pm 4.3$ ).

\section{Instrumentos e Tarefa}

$\mathrm{O}$ instrumento utilizado foi $\mathrm{O}$ Bassin Anticipation Timer (Lafayette Instruments, modelo n. ${ }^{\circ}$ 50575) que consiste numa calha metálica com díodos emissores de luz (LEDs) dispostos em sequência e distanciados $4.5 \mathrm{~cm}$ entre si. As tarefas sincronizadas usadas envolveram diferentes níveis de complexidade motora. Em ambas as tarefas, a calha foi posicionada de frente para o sujeito, de forma que o sinal luminoso se deslocasse da extremidade distal para a proximal.

A tarefa simples consistiu em accionar com o polegar um botão de pressão colocado na mão no momento em que o último LED acendia. A tarefa complexa consistiu em tocar cinco sensores numa sequência prédeterminada $(1,2,3,4,5)$ em integração com um estímulo visual, de forma que o último sensor (5) fosse tocado simultaneamente com a chegada do estímulo luminoso ao último LED. Para tal foi utilizado o aparelho de antecipaçãocoincidência em tarefas complexas (Corrêa, 2001). Na tarefa complexa era o participante que dava início ao deslocamento do estímulo carregando num sensor colocado na sua linha média, perto dos restantes sensores. A calha utilizada para esta tarefa foi a mesma utilizada para a tarefa simples. Acoplados aos sensores encontrava-se um computador com um software que possibilitava o registo automático dos resultados, ou seja o tempo de antecipaçãocoincidência, que se refere ao tempo entre o último toque e o acendimento do LED alvo. Em ambas as tarefas os sujeitos adoptaram a mesma posição (de pé), estando o ângulo de aproximação do estímulo a $30^{\circ}$ (Payne, 1987).

\section{Procedimentos}

Os sujeitos foram informados oralmente dos objectivos do estudo e das tarefas. A amostra foi contrabalançada em relação à variável complexidade da tarefa sendo dividida em dois grupos de forma aleatória.

$\mathrm{O}$ primeiro grupo iniciou as tentativas na tarefa simples e executou depois a tarefa complexa (grupo S-C). O segundo grupo iniciou a tarefa complexa à qual se seguiu a simples (grupo C-S).

Todos os participantes realizaram 5 tentativas com uma mão e 5 tentativas com a outra em cada uma das tarefas, sendo os grupos contrabalançados em relação à mão que iniciava a tarefa. $\mathrm{Na}$ tarefa simples a velocidade utilizada foi de $268.2 \mathrm{~cm} / \mathrm{s}(6 \mathrm{mph}$ ) e na tarefa complexa foi de $44.7 \mathrm{~cm} / \mathrm{s}$ (1 $\mathrm{mph})$. O 
foreperiod, ou seja, o sinal de aviso (díodo amarelo) mantinha-se aceso durante $.5 \mathrm{~s}$ antes do início da propagação do estímulo, em qualquer uma das tarefas. Em todas as etapas foi fornecido conhecimento verbal dos resultados (0 - $25 \mathrm{~ms}$ : Excelente; 26 - $50 \mathrm{~ms}$ : Muito Bom; 51 - 100 ms: Bom; acima dos 101 ms: muito antes ou muito depois). Estas categorias basearam-se nas usadas por Corrêa (2001).

\section{Análise Estatística}

Foram calculados os erros absoluto (EA), constante (EC) e variável (EV) para cada participante. As variáveis dependentes foram analisadas em cada grupo de preferência manual através de uma ANOVA multifactorial $2 \times 2 \times 2 \times 2$ (idade, sexo, mão, tarefa), com medidas repetidas nos dois últimos factores. $\mathrm{O}$ nível de significância foi fixado em $p \leq .05$.

\section{RESULTADOS}

Na Tabela 1 estão descritos, para o grupo de preferência manual, a média e o desvio padrão de cada tipo de erro, relativamente a cada mão, a cada tarefa e considerando a idade e o sexo.

\section{Erro Absoluto}

\section{Destrímanos}

Os factores principais tarefa $\left[F_{(1,55)}=56.88\right.$, $p<.001]$, idade $\left[F_{(1,55)}=15.41, p<.001\right] \mathrm{e}$ sexo $\left[F_{(1,55)}=11.83, p=.001\right]$ tiveram um efeito significativo, assim como a interacção entre os factores tarefa e idade $\left[F_{(1,55)}=6.15, p\right.$ $<.001]$ e entre tarefa e sexo $\left[F_{(1,55)}=4.39, p=\right.$ $.041]$. Os factores principais revelaram uma precisão mais elevada no grupo mais velho, no sexo masculino e na tarefa simples. As duas interacções revelam que a diferença entre as idades e entre os sexos foi superior na tarefa complexa.

Tabela 1

Média, desvio padrão, F e p dos erros absoluto (EA), constante (EC) e variável (EV) em relação à mão preferida (MP) e não preferida (MNP), na tarefa simples e complexa, considerando a idade e o sexo

\begin{tabular}{|c|c|c|c|c|c|c|c|}
\hline \multirow{2}{*}{ Variáveis } & & \multicolumn{2}{|c|}{ EA } & \multicolumn{2}{|c|}{ EC } & \multicolumn{2}{|c|}{ EV } \\
\hline & & Destrímanos & Sinistrómanos & Destrímanos & Sinistrómanos & Destrímanos & Sinistrómanos \\
\hline \multirow[t]{4}{*}{ Mão } & MP & $110.5 \pm 62.1$ & $97.6 \pm 50.2$ & $13.0 \pm 80.2$ & $-1.6 \pm 75.3$ & $105.8 \pm 63.3$ & $91.5 \pm 58.9$ \\
\hline & MNP & $108.4 \pm 62.6$ & $112.9 \pm 67.6$ & $29.4 \pm 79.8$ & $19.7 \pm 100.4$ & $107.2 \pm 68.5$ & $107.3 \pm 60.5$ \\
\hline & $F$ & .087 & 4.322 & 4.457 & 4.547 & .031 & 4.868 \\
\hline & $p$ & .769 & .043 & .039 & .038 & .860 & .032 \\
\hline \multirow[t]{4}{*}{ Tarefa } & Simples & $74.8 \pm 33.3$ & $72.9 \pm 28.5$ & $27.2 \pm 51.5$ & $12.4 \pm 48.0$ & $66.8 \pm 32.5$ & $71.7 \pm 32.1$ \\
\hline & Complexa & $144.1 \pm 91.4$ & $137.6 \pm 87.3$ & $15.2 \pm 108.5$ & $5.7 \pm 127.7$ & $146.1 \pm 99.3$ & $127.1 \pm 87.3$ \\
\hline & $F$ & 54.942 & 69.601 & .908 & .265 & 61.177 & 52.517 \\
\hline & $p$ & $<.001$ & $<.001$ & .345 & .609 & $<.001$ & $<.001$ \\
\hline \multirow[t]{4}{*}{ Idade } & 7-8 anos & $131.8 \pm 66.4$ & $128.3 \pm 69.2$ & $18.5 \pm 100.4$ & $11.1 \pm 109.3$ & $125.3 \pm 68.8$ & $123.4 \pm 68.7$ \\
\hline & 9-10 anos & $87.1 \pm 53.8$ & $82.1 \pm 34.9$ & $23.9 \pm 59.8$ & $7.0 \pm 63.1$ & $87.7 \pm 60.7$ & $75.4 \pm 40.0$ \\
\hline & $F$ & 15.355 & 25.430 & .110 & .046 & 10.759 & 32.574 \\
\hline & $p$ & $<.001$ & $<.001$ & .741 & .830 & .002 & $<.001$ \\
\hline \multirow[t]{4}{*}{ Sexo } & Masculino & $89.9 \pm 57.8$ & $92.2 \pm 43.9$ & $13.2 \pm 77.8$ & $4.1 \pm 79.3$ & $85.1 \pm 58.4$ & $82.7 \pm 42.4$ \\
\hline & Feminino & $129.1 \pm 64.7$ & $118.2 \pm 70.8$ & $29.2 \pm 82.3$ & $14.1 \pm 99.1$ & $127.8 \pm 70.8$ & $116.1 \pm 73.6$ \\
\hline & $F$ & 11.820 & 8.062 & .948 & .277 & 13.836 & 15.820 \\
\hline & $p$ & .001 & .006 & .334 & .601 & $<.001$ & $<.001$ \\
\hline
\end{tabular}




\section{Sinistrómanos}

Os factores principais tarefa $\left[F_{(1,52)}=72.75\right.$, $p<.001]$, mão $\left[F_{(1,52)}=5.94, p=.018\right]$, idade $\left[F_{(1,52)}=23.44, p<.001\right]$ e sexo $\left[F_{(1,52)}=5.22\right.$, $p=.026]$ tiveram um efeito significativo. Uma precisão mais elevada foi observada na tarefa simples, na MP, nos participantes mais velhos e no sexo masculino. Para além disso, a interacção entre os factores tarefa e idade $\left[F_{(1,52)}=10.73, p=.002\right]$ revelou que a diferença entre os grupos de idade foi mais acentuada na tarefa complexa.

\section{Erro Constante}

\section{Destrímanos}

O factor principal mão $\left[F_{(1,55)}=4.86, p=\right.$ .032] e a interacção entre mão e tarefa $\left[F_{(1,55)}=\right.$ 4.98, $p=.030]$ revelaram um efeito significativo. Esta interacção demonstrou que a assimetria manual foi mais elevada na tarefa complexa do que na simples. $\mathrm{Na}$ tarefa complexa, os sujeitos anteciparam as respostas e demonstraram um enviesamento do erro menor com a MP do que com a MNP, com a qual atrasaram as respostas.

\section{Sinistrómanos}

Apenas o factor principal mão $\left[F_{(1,52)}=\right.$ $4.55, p=.038]$ apresentou significado estatístico, demonstrando a MP menor enviesamento do erro e a tendência para respostas antecipadas, relativamente à MNP que demonstrou maior enviesamento do erro e propensão para respostas mais atrasadas.

\section{Erro Variável}

\section{Destrímanos}

Os factores principais tarefa $\left[F_{(1,55)}=63.28\right.$, $p<.001]$, idade $\left[F_{(1,55)}=10.96, p=.002\right] \mathrm{e}$ sexo $\left[F_{(1,55)}=14.10, p<.001\right]$ tiveram um efeito significativo, assim como a interacção entre os factores tarefa e sexo $\left[F_{(1,55)}=6.19, p\right.$ $=.016]$. As crianças mais velhas e o sexo masculino apresentaram menor variabilidade e a interacção revelou que a diferença entre os sexos foi mais elevada na tarefa complexa do que na simples.

\section{Sinistrómanos}

Os factores principais tarefa $\left[F_{(1,52)}=49.48\right.$, $p<.001]$, mão $\left[F_{(1,52)}=6.63, p=.013\right]$, idade $\left[F_{(1,52)}=28.30, p<.001\right]$ e sexo $\left[F_{(1,52)}=9.23\right.$, $p=.004]$ tiveram um efeito significativo, assim como a interacção entre os factores tarefa e idade $\left[F_{(1,52)}=11.62, p=.001\right]$ e entre idade e sexo $\left[F_{(1,52)}=9.76, p=.003\right]$. Os factores principais revelaram uma variabilidade menor na tarefa simples, na MP, no grupo mais velho e no sexo masculino. A primeira interacção revelou que a diferença entre as idades foi maior na tarefa complexa e a segunda interacção demonstrou que a diferença entre os sexos foi mais acentuada no grupo 7-8 anos.

\section{DISCUSSÃO}

O objectivo principal deste estudo consistiu em investigar a assimetria manual em função da complexidade da tarefa, pretendendo ainda analisar o efeito da idade e do sexo nessa mesma assimetria, em crianças destrímanas e sinistrómanas. Os resultados revelaram um aumento da assimetria manual com a complexidade da tarefa apenas na análise do EC. Este aumento da assimetria foi observado tanto nos destrímanos como nos sinistrómanos, apesar de neste grupo a diferença entre mãos não ter atingido significado estatístico, comprovando parcialmente os resultados de outros trabalhos (Borod et al., 1984; Bryden \& Roy, 1999; Bryden et al., 2007; Flowers, 1975; Miller, 1982; Provins \& Magliaro, 1989). A sequência de acções de complexidade de execução mais elevada, requerida pela tarefa complexa comparativamente à tarefa simples, fez realçar a vantagem da MP. Tal como enfatizou Bryden (1998), a superioridade da MP em relação à MNP irá ser evidente em tarefas que requerem características como, por exemplo, complexa sequência de movimentos, orientação precisa da mão, controlo visual online e AC.

Apesar de ambos os grupos não terem sido comparados entre si, foi possível verificar que a assimetria manual foi evidente nos sinistrómanos em todos os erros de medida, 
enquanto nos destrímanos apenas se verificou na análise do EC e durante a execução da tarefa complexa. Estes resultados contrariam os de outros estudos nos quais foi observada uma assimetria manual mais elevada nos destrímanos (Olex-Zarychta \& Raczek, 2008). A natureza da tarefa estará provavelmente associada a este efeito. Por um lado, a percepção da trajectória de um objecto em movimento envolve o processamento visuoespacial; por outro lado, a predição do local e do tempo que o estímulo demora a chegar ao alvo requer estimativas espaço-temporais. Tem sido descrito na literatura que o processamento da informação requerido por este tipo de tarefa pode envolver uma vantagem do hemisfério direito sobre o hemisfério esquerdo (Boulinguez, Ferrois, \& Graumer, 2003; Dane \& Erzurumluoglu, 2003; Gordon \& Kravetz, 1991; Holtzen, 2000), resultando num benefício para os sinistrómanos, uma vez que a sua MP (esquerda) é comandada sobretudo pelo hemisfério direito. Esta situação seria então a causadora de uma assimetria manual superior relativamente aos destrímanos.

No geral, a assimetria manual não variou em função da idade. Porém, quer a análise do EA quer a do EV revelou que em ambos os grupos de PM a idade foi um factor com efeito significativo, sendo o grupo de 9-10 anos mais preciso e menos variável do que o grupo de 7-8 anos. Estes resultados corroboram os de outros estudos (Ball \& Glencross, 1985; Bard, Fleury, Carrière, \& Bellec, 1981; Dorfman, 1977; Stadulis, 1971), que explicam esta observação sugerindo uma integração sensório-motora mais desenvolvida no grupo mais velho permitindo, desta forma, um desempenho mais proficiente. Tem sido sugerido que a experiência e a aprendizagem adicional na intercepção de estímulos nas crianças mais velhas possam superar as qualidades perceptivas ou motoras necessárias à realização deste tipo de tarefas. As crianças mais velhas parecem recorrer a um número mais elevado de pistas e de elementos de análise na intercepção de trajectórias de estímulos visuais
(Stadulis, 1985). A interacção da idade com a tarefa revelou um melhor desempenho dos mais velhos na execução de tarefas complexas. Estes resultados corroboram outros estudos onde estas variáveis foram analisadas (Fleury \& Bard, 1985). As tarefas complexas parecem assim determinar um peso atencional diferenciado em função da idade, em adição ao processamento da informação (Bard et al., 1990).

O efeito do sexo foi visível em ambos os grupos de preferência manual, revelando o sexo masculino uma melhor performance do que o sexo feminino. Este resultado contraria vários estudos nos quais não se observaram diferenças entre os sexos em participantes prépúberes na realização de tarefas de AC (Millslagle, 2004; Ridenour, 1981; Wrisberg \& Mead, 1983). Porém, um facto interessante merece destaque, podendo explicar a ausência de diferenças entre os sexos nos estudos atrás citados: estes recorreram apenas à aplicação de tarefas simples. No presente estudo foi observado que o efeito do sexo está relacionado com a complexidade da tarefa, corroborando outros estudos (Fleury \& Bard, 1985). Habilidades visuo-espaciais mais proficientes no sexo masculino podem ser apontadas como uma das possíveis causas destes resultados (Bell, Willson, Wilman, Dave, \& Silverstone, 2006; Dane \& Erzurumluoglu, 2003).

A tarefa simples proporcionou um desempenho mais elevado do que a tarefa complexa em ambos os grupos de PM, indo ao encontro dos resultados de outras investigações que avaliaram o efeito da complexidade em tarefas de AC (Williams, Jasiewicz, \& Simmons, 2001; Williams \& Jasiewicz, 2001). Este resultado era esperado, uma vez que o desempenho em tarefas complexas requer planeamento e organização do movimento e, consequentemente, maior demanda na programação motora.

\section{CONCLUSÕES}

Os resultados deste estudo revelaram (i) um aumento da assimetria manual com a complexidade da tarefa em ambos os grupos de 
PM, apesar de nos sinistrómanos a diferença entre as mãos não ter atingido significado estatístico; (ii) uma assimetria manual em todos os erros de medida, evidente apenas nos sinistrómanos; (iii) maior precisão e menor variabilidade do grupo de 9-10 anos comparativamente ao grupo de 7-8 anos, em ambos os grupos de PM, revelando a interacção da idade com a tarefa que este efeito maturacional foi mais pronunciado na execução de tarefas complexas; (iv) um desempenho mais elevado do sexo masculino em relação ao sexo feminino em ambos os grupos de PM; (v) um desempenho mais elevado na tarefa simples comparativamente à tarefa complexa em ambos os grupos de PM. Estes resultados favorecem a perspectiva na qual a assimetria manual é considerada como um processo dinâmico, interagindo com o tipo de tarefa, com a idade e o sexo. Demonstram, ainda, que os dois grupos de preferência manual não se comportam da mesma forma no desempenho da capacidade de AC, traduzida no presente estudo em duas tarefas de diferente complexidade.

\section{REFERÊNCIAS}

Amunts, K., Jancke, L., Mohlberg, H., Steinmetz, H., \& Zilles, K. (2000). Interhemispheric asymmetry of the human motor cortex related to handedness and gender. Neuropsychologia, 38(3), 304-312.

Ball, C. T., \& Glencross, D. (1985). Developmental differences in a coincident timing task under speed and time constraints. Human Movement Science, 4(1), 1-15.

Bard, C., Fleury, M., Carrière, L., \& Bellec, J. (1981). Components of the coincidence-anticipation behavior of children aged from 6 to 11 years. Perceptual and Motor Skills, 52 (2), 547-556.

Bard, C., Fleury, M., \& Gagnon, M. (1990). Coincidence-anticipation timing: An age related perspective. In C. Bard, M. Fleury, \& L. Hay (Eds.), Development of eye-hand coordination across life span (pp. 283-305). Columbia: University of South Carolina Press.

Bell, E. C., Willson, M. C., Wilman, A. H., Dave, S., \& Silverstone, P. H. (2006). Males and females differ in brain activation during cognitive tasks. Neuroimage, 30(2), 529-538.
Borod, J. C., Caron, H. S., \& Koff, E. (1984). Lefthanders and right-handers compared on performance and preference measures of lateral dominance. British Journal of Psychology, 75(Pt 2), 177-186.

Boulinguez, P., Ferrois, M., \& Graumer, G. (2003). Hemispheric asymmetry for trajectory perception. Cognitive Brain Research, 16(2), 219-225.

Bryden, M. P., \& Mayer, M. (2008). Hand preference and performance abilities in children and adults. Brain and Cognition, 67(S1), S15.

Bryden, P. (1998). The origins of manual asymmetries: What is revealed by pushing the limits of task difficulty. Unpublished Doctoral Thesis, University of Waterloo, Waterloo, Ontario, Canada.

Bryden, P. J. (2002). Pushing the limits of task difficulty for the right and left hands in manual aiming. Brain and Cognition, 48(2/3), 287-291.

Bryden, P. J., \& Roy, E. A. (1999). Spatial task demands affect the extent of manual asymmetries. Laterality, 4(1), 27-37.

Bryden, P. J., Roy, E. A., Rohr, L. E., \& Egilo, S. (2007). Task demands affect manual asymmetries in pegboard performance. Laterality, 12 (4), 364-377.

Bryden, P. J., Roy, E. A., \& Spence, J. (2007). An observational method of assessing handedness in children and adults. Developmental Neuropsychology, 32 (3), 825-846.

Cockerill, I. M., Van-Zyl, P. A., \& Nevill, A. M. (1988). Functional asymmetry and the development of anticipation-timing. The Physical Education Association Research, S3, 7-10.

Coker, C. (2004). Bilateral symmetry in coincident timing: A preliminary investigation. Perceptual and Motor Skills, 98(1), 359-365.

Corrêa, U. (2001). Estrutura de prática e processo adaptativo na aquisição de habilidades motoras (Tese de Doutorado). Universidade de São Paulo, São Paulo, Brasil.

Dane, S., \& Erzurumluoglu, A. (2003). Sex and handedness differences in eye-hand visual reaction times in handball players. International Journal of Neuroscience, 113(7), 923-929.

Dellatolas, G., De Agostini, M., Curt, F., Kremin, H., Letierce, A., Maccario, J., \& Lellouch, J. (2003). Manual skill, hand skill asymmetry, and cognitive performances in young children. Laterality, 8(4), 317-338.

Dorfman, P. (1977). Timing and anticipation: A developmental perspective. Journal of Motor Behavior, 9(1), 67-79. 
Elalmis, D. D., \& Tan, U. (2008). Dynamics of manual skill: A computerized analysis of single peg movements and stochastic resonance hypothesis of cerebral laterality. International Journal of Neuroscience, 118(3), 399-432.

Fearing, M. K., Browning, C. A., Corey, D. M., \& Foundas, A. L. (2001). Dual-task performance in right- and left-handed adults: A fingertapping and foot-tapping study. Perceptual and Motor Skills, 92 (2), 323-334.

Fleury, M., \& Bard, C. (1985). Age, stimulus velocity and task complexity as determiners of coincident timing behavior. Journal of Human Movement Studies, 11, 305-317.

Flowers, K. (1975). Handedness and controlled movement. British Journal of Psychology, 66(1), 39-52.

Gordon, H. W., \& Kravetz, S. (1991). The influence of gender, handedness, and performance level on specialized cognitive functioning. Brain and Cognition, 15(1), 37-61.

Gurd, J. M., Schulz, J., Cherkas, L., \& Ebers, G. C. (2006). Hand preference and performance in 20 pairs of monozygotic twins with discordant handedness. Cortex, 42 (6), 934-945.

Haaland, K. Y., \& Harrington, D. L. (1996). Hemispheric asymmetry of movement. Current Opinion in Neurobiology, 6(6), 796-800.

Herve, P. Y., Leonard, G., Perron, M., Pike, B., Pitiot, A., Richer, L., ... Paus, T. (2009). Handedness, motor skills and maturation of the corticospinal tract in the adolescent brain. Human Brain Mapping, 30(10), 3151-3162.

Herve, P. Y., Mazoyer, B., Crivello, F., Perchey, G., \& Tzourio-Mazoyer, N. (2005). Finger tapping, handedness and grey matter amount in the Rolando's genu area. Neuroimage, 25(4), 11331145.

Holtzen, D. (2000). Handedness and professional tennis. International Journal of Neuroscience, 105(1/4), 101-119.

Kumar, S., \& Mandal, M. K. (2004). Motor performance as a function of verbal, nonverbal interference and handedness. International Journal of Neuroscience, 114(7), 787-794.

Lage, G. M., Gallo, L. G., Miranda, M. G., Vieira, D. R., Schickler, D. J., Coelho, R. R., ... Benda, R. (2008). Assimetrias manuais e complexidade da tarefa em habilidades de apontamento. Revista Portuguesa de Ciências do Desporto, 8(1), 47-57.
Les, W. R., Katene, W. H., \& Fleming, K. (2002). Coincidence timing of a tennis stroke: Effects of age, skill level, gender, stimulus velocity, and attention demand. Research Quarterly for Exercise \& Sport, 73(1), 28-37.

McManus, C., Sik, G., Cole, D. R., Mellon, A. F., Wong, J., \& Kloss, J. (1988). The development of handedness in children. British Journal of Developmental Psychology, 6, 257-273.

Miller, C. A. (1982). Degree of lateralization as a hierarchy of manual and cognitive skill levels. Neuropsychologia, 20(2), 155-162.

Millslagle, D. (2004). Coincidence anticipation and dynamic visual acuity in young adolescents. Perceptual and Motor Skills, 99(3 Pt 2), 11471156.

Nalcaci, E., Kalaycioglu, C., Cicek, M., \& Genc, Y. (2001). The relationship between handedness and fine motor performance. Cortex, 37(4), 493-500.

Olex-Zarychta, D., \& Raczek, J. (2008). The relationship of movement time to hand-foot laterality patterns. Laterality, 13(5), 439-455.

Pascual-Leone, A., \& Torres, F. (1993). Plasticity of the sensorimotor cortex representation of the reading finger in Braille readers. Brain, $116(\mathrm{Pt}$ 1), 39-52.

Payne, V. G. (1987). Effects of angle of stimulus approach on coincidence-anticipation timing performance. Journal of Human Movement Studies, $13,383-390$

Provins, K. A. (1997). The specificity of motor skill and manual asymmetry: A review of the evidence and its implications. Journal of Motor Behavior, 29(2), 183-192.

Provins, K. A., \& Magliaro, J. (1989). Skill, strength, handedness, and fatigue. Journal of Motor Behavior, 21 (2), 113-121.

Ridenour, M. V. (1981). Influence of background patterns of coincidence-anticipation performance. Perceptual and Motor Skills, 52 (1), 47-50.

Rodrigues, P., Vasconcelos, O., Barreiros, J., \& Barbosa, R. (2009). Manual asymmetry in a complex coincidence-anticipation task: Handedness and gender effects. Laterality, 14(4), 395-412.

Rodrigues, P., Vasconcelos, O., Barreiros, J., Barbosa, R., \& Trifílio, F. (2009). Functional asymmetry in a simple coincidence-anticipation task: Effects of handedness. European Journal of Sport Science, 9(2), 115-123. 
Shen, Y. C., \& Franz, E. A. (2005). Hemispheric competition in left-handers on bimanual reaction time tasks. Journal of Motor Behavior, 37(1), 3-9.

Stadulis, R. E. (1971). Coincidence-anticipation behavior of children (Unpublished doctoral dissertation). Columbia University, New York, USA.

Stadulis, R. E. (1985). Coincidence-anticipation behavior of children. In J. E. Clark \& J. H. Humphrey (Eds.), Motor development (Vol. 1, pp. 1-17). Princeton: Princeton Book Company.

Steenhuis, R. E. (1999). The relation between hand preference and hand performance: What you get depends on what you measure. Laterality, 4(1), 3-26.

Teixeira, L. A., Gasparetto, E. R., \& Sugie, M. M. (1999). Is there manual asymmetry in movement preparation? Perceptual and Motor Skills, 89(1), 205-208.

Teixeira, L. A., \& Paroli, R. (2000). Assimetrias laterais em acções motoras: Preferência versus desempenho. Motriz, 6(1), 1-8.

Triggs, W. J., Calvanio, R., \& Levine, M. (1997). Transcranial magnetic stimulation reveals a hemispheric asymmetry correlate of intermanual differences in motor performance. Neuropsychologia, 35(10), 1355-1363.

Van Strien, J. W. (1992). Classificatie van links - en rechtshangige proefperrsonen [Classification of left- and right-handed research participants]. Nederlands Tijdschrift voor de Psychologie, 47, 8892.

Volkmann, J., Schnitzler, A., Witte, O. W., \& Freund, H. (1998). Handedness and asymmetry of hand representation in human motor cortex. Journal of Neurophysiology, 79(4), 2149-2154.

Williams, L., Jasiewicz, J., \& Simmons, R. (2001). Coincidence timing of finger, arm, and whole body movements. Perceptual and Motor Skills, $92(2), 535-547$.

Williams, L., \& Jasiewicz, J. M. (2001). Knowledge of results, movement type, and sex in coincidence timing. Perceptual and Motor Skills, 92 (3 Pt 2), 1057-1068.

Wrisberg, C. A., \& Mead, B. J. (1983). Developing coincident timing skill in children: A comparison of training methods. Research Quarterly for Exercise and Sport, 54(1), 67-74.

(c)) EY-No Todo o conteúdo da revista Motricidade está licenciado sob a Creative Commons, excepto quando especificado em contrário e nos conteúdos retirados de outras fontes bibliográficas. 